\title{
Visuotactile interactions in the congenitally acallosal brain: Evidence for early cerebral plasticity
}

\author{
Claudia C. Wolfa, ${ }^{a}$, Anna Balla ${ }^{\mathrm{a}}$, Sebastian Ocklenburg ${ }^{\mathrm{a}}$, Tobias Otto ${ }^{\mathrm{a}}$, Tobias Heed $^{\mathrm{b}}$, \\ Brigitte Röder ${ }^{\mathrm{b}}$, Onur Güntürkün ${ }^{\mathrm{a}}$ \\ ${ }^{a}$ Ruhr-University Bochum, Department of Biopsychology, Universitätsstraße 150, 44780 Bochum, Germany \\ ${ }^{\mathrm{b}}$ University of Hamburg, Biological Psychology and Neuropsychology, Von-Melle-Park 11, 20146 Hamburg, Germany
}

\section{A R T I C L E I N F O}

\section{Article history:}

Received 15 June 2011

Received in revised form

21 September 2011

Accepted 7 October 2011

Available online 14 October 2011

\section{Keywords:}

Agenesis of the corpus callosum

Crossmodal congruency task

Interhemispheric interaction

Multisensory

Neuronal plasticity

Visuotactile

\begin{abstract}
A B S T R A C T
Studies in patients with an isolated, congenital agenesis of the corpus callosum have documented potentials and limits of brain plasticity. Literature suggests that early reorganization mechanisms can compensate for the absence of the corpus callosum in unisensory tasks that involve interhemispheric transfer. It is unknown, however, how the congenitally acallosal brain processes multisensory information, which presumably requires interhemispheric transfer of modality-specific input. Therefore, we tested five patients with total and one patient with partial agenesis of the corpus callosum in a visuotactile interference task (the "crossmodal congruency task") with uncrossed and crossed hands and compared their performance to that of 31 healthy controls. We found that congruency effects followed the hands in space not only in healthy, but also in congenitally acallosal individuals. Remarkably, this was also true when patients' hands crossed the vertical visual meridian and stimuli were presented at the same hand. These results suggest that callosal connectivity is not required for remapping of visuotactile space. We conclude that early brain plasticity allows for compensation of the developmental absence of the corpus callosum in a visuotactile interference task.
\end{abstract}

(C) 2011 Elsevier Ltd. All rights reserved.

\section{Introduction}

Plasticity is a fundamental principle of brain organization (Duffau, 2006; Kleim \& Jones, 2008; Kolb, Gibb, \& Robinson, 2003). Neural remodelling mechanisms allow for an environmental adaptation and acquisition of new skills, but also for recovery from brain damage and coping with congenital malformations. Although the structure and function of the brain are modified throughout life (Bavelier, Levi, Li, Dan, \& Hensch, 2010; Gage, 2004; Ramachandran, 1993), the adaptive capacity is considerably higher in the developing compared to the adult brain (Bavelier et al., 2010; Johnston, 2004; Johnston et al., 2009).

Evidence for this prominence of early reorganization is given by functional differences between split brain patients and patients with an isolated, congenital agenesis of the corpus callosum (AgCC). Both groups have in common the total absence of callosal connectivity. In healthy individuals, the corpus callosum connects homologous cortical areas through 200-350 million nerve fibres (Aboitiz, Scheibel, Fisher, \& Zaidel, 1992a; Aboitiz, Scheibel, Fisher, \& Zaidel, 1992b), and can have both inhibitory and excitatory influences on the contralateral hemisphere (Bloom \& Hynd, 2005).

\footnotetext{
* Corresponding author. Tel.: +49 2343226804; fax: +49 2343214377. E-mail address: claudia.c.wolf@ruhr-uni-bochum.de (C.C. Wolf).
}

However, whereas the corpus callosum of split brain patients was severed in a surgical commissurotomy during later life to treat otherwise intractable epilepsy (reviewed by Gazzaniga, 1995, 2005), patients with AgCC suffer from a congenital absence of the corpus callosum (reviewed by Paul et al., 2007). Typically, genetic factors give rise to AgCC (Bedeschi et al., 2006; Schell-Apacik et al. 2008). Nevertheless, environmental influences can also contribute to a disruption in any of the multiple steps involved in callosal development (Guerri, Pascual, \& Renau-Piqueras, 2001; Riley et al., 1995).

Functional differences between split brain and AgCC patients have been documented in tasks that involve interhemispheric processing of unisensory, simple, and familiar information (Paul et al., 2007). In such tasks, performance of AgCC patients is either comparable to that of healthy individuals or lies between healthy and split brain individuals. Basically, the classical "disconnection syndrome", the complete absence of interhemispheric transfer of information derived from a stimulus presented unilaterally (Chiarello, 1980; Seymour, Reuter-Lorenz, \& Gazzaniga, 1994; Sperry, Gazzaniga, \& Bogen, 1969), is only found in the split but not in the congenitally acallosal brain (Lassonde, Sauerwein, Chicoine, \& Geoffroy, 1991). The presence of interhemispheric crosstalk in patients with $\mathrm{AgCC}$ is supported by an intact interhemispheric Stroop interference effect (Brown, Thrasher, \& Paul, 2001), and a typical bilateral field advantage for the comparison of simple visual information 
across hemifields (Brown, Jeeves, Dietrich, \& Burnison, 1999). Moreover, it was found that AgCC patients do not differ from healthy controls in transfer tasks that involve bimanual integration of kinesthetic, somesthetic, and motor functions, although acallosal patients were impaired with respect to speed (Sauerwein, Lassonde, Cardu, \& Geoffroy, 1981). Decreased reaction times are also found in behavioural estimates of interhemispheric transfer time as measured with the Poffenberger paradigm (Poffenberger, 1912), in which participants have to respond as fast as possible to visual cues. Here, prolonged transfer times in AgCC patients compared to healthy individuals are demonstrated, although AgCC patients transfer information still more rapidly than split brain patients (Mooshagian, Iacoboni, \& Zaidel, 2009).

Possibly, the preserved capacity for interhemispheric transfer of unisensory, simple or familiar information in AgCC patients is due to an early onset of compensatory mechanisms in cross-cortical pathways that remain intact, e.g. the anterior commissure, a small band of axons that connects the temporal lobes (Di Virgilio, Clarke, Pizzolato, \& Schaffner, 1999; Klinger \& Gloor, 1960). Whereas all cerebral commissures including the anterior commissure are severed in the split brain, the anterior commissure tends to be intact in AgCC patients and is even enlarged in several cases (Hetts, Sherr, Gobuty, Chao, \& Barkovich, 2006).

That compensatory mechanisms are indeed most powerful during early development was demonstrated by Lassonde, Sauerwein, McCabe, Laurencelle, and Geoffroy (1988), who investigated performance of congenitally acallosal and callosotomized patients in tasks that involved inter- and intrahemispheric comparisons of visual and tactile stimuli. These authors found that older callosotomized children showed disconnection deficits similar to those reported in adult split brain patients, whereas AgCC patients and a very young patient with complete callosal transection demonstrated a high level of accuracy in an interhemispheric tasks. This suggested that compensatory mechanisms may also become manifest in the split brain, when the corpus callosum is surgically disconnected during early life.

Nevertheless, other evidence suggests a limit to the brain's ability to compensate for the absence of the corpus callosum which becomes evident in tasks with increased requirements (Paul et al., 2007). For instance, AgCC patients perform considerably worse when comparing briefly presented stimuli that are unfamiliar, or difficult to verbalize (Brown et al., 1999). Similarly, children with callosal absence also show deficits in bimanual texture matching tasks, likely due to the fact that this task involves refined spatial interpretation of somatosensory input (Friefeld, MacGregor, Chuang, \& Saint-Cyr, 2000). Furthermore, in a computerized version of the bimanual coordination test, which measures bimanual performance over a wide range of tasks, AgCC patients performed significantly slower and less accurate than controls (Mueller, Marion, Paul, \& Brown, 2009). An investigation of bimanual coordination in the Preilowski task suggests that fast and coordinated performance on bimanual tasks depend on the anterior portion of the corpus callosum (Jeeves, Silver, \& Jacobson, 1988; Silver \& Jeeves, 1994). Hines, Paul, and Brown (2002) investigated spatial attention in AgCC patients and healthy controls and found that congenital absence of the corpus callosum reduced the efficiency to reorient attention between visual fields.

Until now, however, research in AgCC patients has focussed exclusively on unisensory tasks, but has neglected multisensory processing. Multisensory tasks differ from unisensory tasks in important aspects given that crossmodal integration is assumed to require transfer of modality-specific information across hemispheres, particularly because sensory cues are coded using different frames of reference at in cortex (McGuire \& Sabes, 2009; Morrell, 1972; Röder, Kusmierek, Spence, \& Schicke, 2007). In order to combine these inputs into a unified percept they are assumed to be recorded in a spatial frame of reference accessible by all sensory modalities (Driver \& Noesselt, 2008). For example, when holding one's right hand in the left part of space, visual information about this hand will arrive in the right visual cortex, while tactile information will arrive in the left somatosensory cortex, necessitating a transfer of information between hemispheres to combine these pieces of information from the different modalities into a common percept. Such recoding of spatial information into a common reference frame is referred to as "spatial remapping".

Although not yet investigated in AgCC patients, multisensory interactions were examined in a split brain patient who underwent a section of corpus callosum at the age of 25 using the crossmodal congruency task (Spence, Kingstone, Shore, \& Gazzaniga, 2001; Spence, Shore, Gazzaniga, Soto-Faraco, \& Kingstone, 2001), which assesses the impact of visual cues on the localization of touch (Heed, Habets, Sebanz, \& Knoblich, 2010; Maravita, Spence, Kennett, \& Driver, 2002; Pavani, Spence, \& Driver, 2000; Schicke, Bauer, \& Röder, 2009; Spence, Pavani, \& Driver, 1998; Spence, Pavani, \& Driver, 2004). The crossmodal congruency task involves speeded tactile discriminations: during each trial, participants have to decide whether a tactile stimulus is presented at the index finger ("above") or thumb ("below") of either hand. Tactile targets are accompanied by visual distractors, which are presented simultaneously, but at independent locations, and are to be ignored by participants. Despite this instruction, discriminations are typically more rapid and accurate when the elevation of the visual distractor is congruent (tactile target and visual distractor both either "above" or "below") than when it is incongruent with the tactile target (tactile target "above" and visual distractor "below" or vice versa). It is assumed that this effect is due to an activation of a hand's somatosensory representation by a visual cue near the hand, thereby causing interference when visual and tactile stimuli are presented at different elevations (Pellegrino, Làvadas, \& Farné, 1997). This process is influenced by spatial proximity: The closer distractors are located to tactile targets in external space (e.g. stimuli at the same hand vs. stimuli at different hands), the larger their impact. These findings of an influence of spatially proximate visual events on touch are thought to indicate automatic spatial remapping of tactile information. The visual-tactile effect of the crossmodal congruency task is usually rather large (in the order of $60-150 \mathrm{~ms}$ ) and very reliable, making this task a prime paradigm to test multisensory processing in patients.

The crossmodal congruency task involves trials with uncrossed and crossed hands. With uncrossed hands, the left hemisphere receives visual input from the right visual half-field and tactile input from the right hand. Comparably, the right hemisphere receives visual input from the left visual half-field and tactile input from the left hand. Therefore, tactile and visual cues that are presented at the same hand presumably map onto the same hemisphere, whereas tactile and visual cues that are presented at different hands presumably map onto different hemispheres. However, in trials in which hands are crossed over the vertical visual meridian, each hand is located in the contralateral visual half-field. In this case, the relationship between visual and tactile information is reversed: Visual stimuli presented to the same hand as the tactile stimulus presumably map onto different hemispheres, whereas visual stimuli presented to the other hand than the tactile stimulus presumably map onto the same hemisphere. For instance, a tactile cue at the right hand maps onto the left hemisphere, but a visual cue near the right hand maps onto the right hemisphere. Thus, the fact that tactile stimuli are automatically remapped to be matched with visual input implies that information is exchanged between hemispheres when the hands are crossed. In other words, when the hands are uncrossed, visual and tactile information from one hemispace arrive in the same hemisphere; in contrast, when 
hands are crossed, visual and tactile information arrive in opposite hemispheres.

In healthy individuals, visuotactile spatial remapping has been found to be independent of whether cues are presented on the same or on different sides of the visual midline (Spence et al., 1998; Spence, Kingstone, et al., 2001; Spence et al., 2004). In a split brain patient, however, remapping was found to be disrupted when cues were presented at the same hand, but the hand crossed the vertical visual meridian (Spence, Kingstone, et al., 2001; Spence, Shore, et al., 2001). Spence, Kingstone, et al. (2001); Spence, Shore, et al. (2001) concluded that callosal connectivity is required for intact processing of visuotactile spatial information.

It is questionable, however, whether this conclusion is applicable to AgCC patients. Possibly, compensatory mechanisms in intact commissures such as the anterior commissure allow for intact remapping of visuotactile information even when spatially proximate cues that are presented at the same hand map onto opposite hemispheres, as is the case in crossed-hand trials. However, the anterior commissure typically connects only frontal and temporal regions of the brain. Therefore, major anatomical changes would be necessary in order to connect regions associated with visuospatial and somatosensory processing. Thus, we investigated remapping of visuotactile space in AgCC patients by means of the crossmodal congruency task and compared their performance to healthy controls. If patients require the corpus callosum for remapping modality-specific spatial representations into a supramodal spatial representation, we would expect a breakdown of the remapping process in trials which require transfer between the hemispheres. However, if the absence of callosal fibres can be compensated for by commissures that remain intact, no difference should exist between individuals with AgCC and healthy controls.

It is well-known that developmental malformations may affect cerebral functioning in several ways (Cao, Vikingstad, Huttenlocher, Towle, \& Levin, 1994; Hicks \& D‘Amato, 1970). The crossmodal congruency task also allowed us to compare performance between AgCC patients and controls for cases in which no hemispheric transfer was necessary to test whether the absence of the corpus callosum alters information processing within one hemisphere.

Taken together, our experimental setup was designed to investigate both same and different hemispace trials with uncrossed and crossed hands, and to draw conclusions about the involvement of callosal connectivity in remapping of visuotactile space.

\section{Methods}

\subsection{Participants}

Four patients with total (B.M., C.F., M.K., M.H.) and one patient with partial AgCC (P.N.) as well as 31 healthy, male controls participated in the present study. Magnetic resonance imaging (MRI) or computed tomography (CT) scans of all patients were available and re-evaluated by a professional neurologist after testing.

The age of patients ranged between 16 and $52(M=29.67, \mathrm{SE}=19.50)$, the age of controls was between 19 and $52(M=27.74, \mathrm{SE}=1.48)$. Mean age did not differ significantly between patients and controls (age: $U=69, p=0.31$, Mann-Whitney $U$-Test, one-tailed).

Patients and controls were native German speakers, had grown up in German middle-class families, and had received mainstream education. Furthermore, they had normal or corrected vision, were naive of the experimental hypothesis, and were paid for participation. All participants gave written informed consent and were treated in accordance with the declaration of Helsinki. The study had been approved by the ethics committee of the Ruhr-University Bochum.

\subsubsection{B.M.}

B.M. developed normally. At the age of 16, the pathology of the corpus callosum was detected incidentally during CT of the nasal synus. Subsequently, MRI was performed, and hypoplasia or total agenesis was diagnosed, accompanied by an enlarged ventricular system. To gain certainty, MRI scans were re-evaluated by a neurologist, who diagnosed an isolated, total AgCC (see Fig. 1a). B.M. had a prominent anterior commissure. Prior to testing, we determined B.M.'s handedness with the Edinburgh Handedness Inventory (Oldfield, 1971). According to the method of
Oldfield, laterality quotients (LQs) ranging between and 100 (complete lefthandedness) and +100 (complete right-handedness) are calculated, with value around 0 indicating ambidextrality. B.M. had an LQ of 33, indicating righthandedness close to ambidextrality. Furthermore, B.M.'s intelligence quotient (IQ) was determined with the Multiple Choice Intelligence Test (Lehrl, 1977), a test routinely used in Germany. B.M.'s IQ was 100 and thus in the average range. During time of testing, B.M. was 21 years old and working in an executive position at an advertising agency.

\subsubsection{M.K}

In M.K., an enlargement of ventricles was detected with prenatal ultrasound recording. Directly after birth, a total AgCC without further malformations was diagnosed on the basis of MRI. Development of M.K.'s language and social communication skills were delayed, which was diagnosed by an educational psychologist When he was 8, dyslexia was diagnosed, whereupon targeted interventions were resorted to. At the age of 15 , the former diagnosis of an isolated, total agenesis was confirmed by CT (see Fig. 1b) and a normally sized anterior commissure was diagnosed. M.K. had an LQ of 37, indicating right-handedness close to ambidextrality. M.K.'s IQ was in the average range (IQ=101). During time of testing, M.K. was 16 years old, had almost finished school, and was in search of an apprenticeship training position.

\subsubsection{P.N.}

The pathology P.N.'s corpus callosum was diagnosed incidentally during MRI at the age of 48. In contrast to B.M. and M.K., P.M. had a partial AgCC: the anterior portion of the corpus callosum was preserved, but Genu, Splenium and Truncus were absent (see Fig. 1c). Like B.M., P.N. had a prominent anterior commissure. The Edinburgh Handedness Inventory revealed an LQ of 19, indicating right-handedness close to ambidextrality. P.N.'s IQ was in the average range $(I Q=100)$. During time of testing, P.N. was 52 years old and was working as an optician.

\subsubsection{C.F.}

The pathology of C.F.'s corpus callosum was diagnosed at the age of six by CT. At this time, C.F. was in a child and adolescent psychiatric clinic in order to examine school aptitude, since his parents had observed severe problems in motor coordination and acquisition of language. He started school at the age of seven, and graduated after six years primary, and six years secondary school. C.F. had a complete agenesis of the corpus callosum AgCC (see Fig. 1d), and a prominent anterior commissure. Furthermore, he had an LQ of -100 , indicating complete left-handedness, and an average $\mathrm{IQ}(\mathrm{IQ}=100)$. During time of testing, C.F. was 23 years old and doing a care assistant (care for the elderly) traineeship programme.

\subsubsection{M.H.}

In M.H., callosal agenesis was diagnosed at the age of 42. During this time, M.H. underwent an in-patient treatment in a secure environment, which was required after an attempted suicide. During treatment, a severe clinical depression and a personality disorder was diagnosed. Furthermore, the pathology of M.H.'s corpus callosum was detected by CT. M.H. suffered from a total AgCC (see Fig. 1e), and had a prominent anterior commissure. Further malformations were diagnosed but not specified by the physician. M.H.'s LQ was 50 , and her IQ was in the average range $(\mathrm{IQ}=104)$. During time of testing, M.H. was 42 years old and out of engagement. Her attending physician accompanied her visit to the Ruhr-University.

\subsection{The crossmodal congruency task}

\subsubsection{Experimental design}

Interactions between vision and touch were examined using the crossmodal congruency task with uncrossed (see Fig. 2a) and crossed hands (see Fig. 2b). The experimental design was modified from Spence et al. (2004). During the task, participants were sitting in a darkened room and focused on a central fixation cross displayed on a computer monitor at a distance of $70 \mathrm{~cm}$. Head movements were minimized by use of an adjustable chinrest. An adjustable armrest was arranged at a distance of $45 \mathrm{~cm}$ from the computer monitor and allowed for a comfort able hand position at eye-level. The distance between hands was $40 \mathrm{~cm}$, both in the uncrossed and crossed hands position. Between index finger and thumb of each hand, participants held foam blocks $(6 \times 6 \times 8 \mathrm{~cm})$, each equipped with two vibrotactile stimulators (Oticon bone conduction vibrators, BC462 100; arranged below finger pads and driven by a $200 \mathrm{~Hz}$ sine wave signal), and two red, lightemitting diodes (Vishay Telefunken LEDs TLHR 4405, luminous intensity $I_{V}=10 \mathrm{mcd}$ arranged beside vibrators)

Prior to the experiment, participants were instructed to focus on the central fixation cross and judge the elevation of vibrotactile targets while simultaneously ignoring the visual distractors. Visuotactile stimulation occurred either congruent (tactile target and visual distractor at same elevation) or incongruent (tactile target and visual distractor at different elevations). Responses occurred as fast and accurate as possible with two foot pedals (Thomann Lead Foot LFD-1), one located beneath the heel, the other beneath the toes of the right foot. Participants lifted their heel to indicate a target at a "lower" position (at the thumb of either hand), and their toes to indicate a target at an "upper" position (at the index finger of eithe 

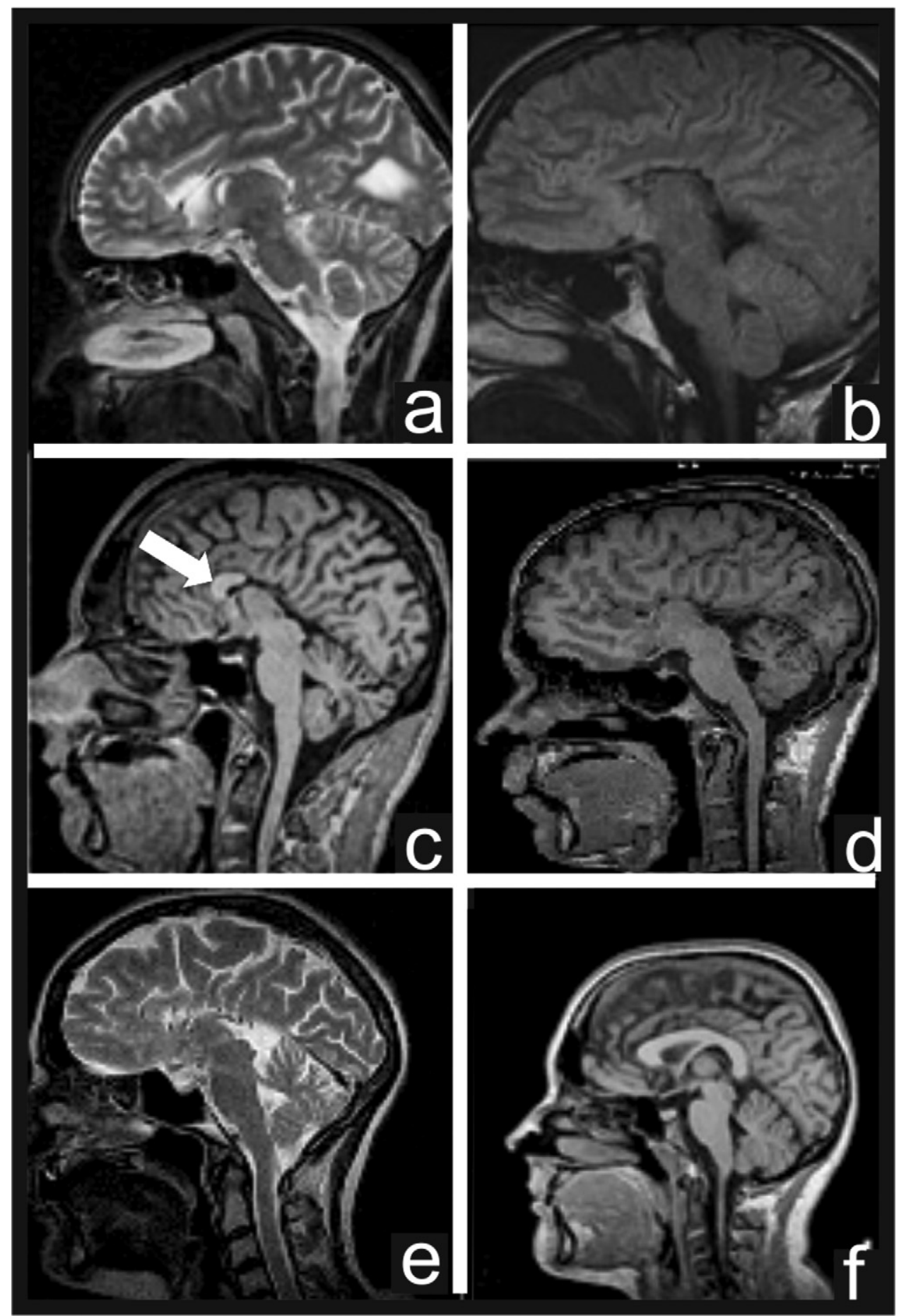

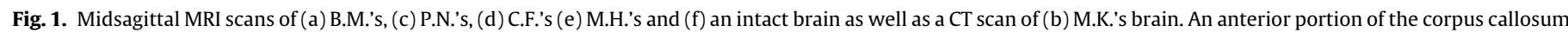
is visible in (c).

hand). Thus, elevation discrimination was independent of the side from which stimuli were presented. If no response had occurred within $1.5 \mathrm{~s}$ after stimulation, the trial was terminated. Otherwise, a trial ended with the participant's response. Each trial consisted of three $50 \mathrm{~ms}$ bursts of vibrotactile stimulations that were separated by 50 ms empty intervals. Tactile stimulations were accompanied by visual stimulations (50 ms light bursts delivered from an LED), which occurred simultaneously, but at independent locations.

Overall, two training blocks (64 trials each) and eight experimental blocks (32 trials each) were conducted. Both training and experimental blocks started with uncrossed hands. Hand posture was changed after each block. Thus, half of the trials were conducted with uncrossed hands, and the other half with crossed hands.

\subsubsection{Data analysis}

We calculated mean error rates, reaction times, and inverse efficiency (IE) of patients and controls. The IE is calculated as average reaction time divided by the percentage of correct responses, and thus attempts to control for speed-accuracy trade-offs (Spence, Kingstone, et al., 2001; Spence, Shore, et al., 2001). For both uncrossed and crossed trial blocks, we compared patients with controls for (1) incongruent same hemispace trials, (2) congruent same hemispace trials, (3) incongruent different hemispace trials, and (4) congruent different hemispace trials, using non-parametric statistics (Mann-Whitney U-Test, two-tailed).

Similarly to previous studies (Spence, Kingstone, et al., 2001; Spence, Shore et al., 2001), we then calculated crossmodal congruency effects (CCEs; performance on incongruent trials minus performance on congruent trials), measured in terms of 
IE. The CCE is an indicator of the influence of visual distractors on touch: a large CCE indicates strong crossmodal modulation of tactile location judgements. First, mean CCEs of healthy controls for same and different hemispace trials in the uncrossed and crossed hands position, respectively, were compared in a paired $t$-test (two-tailed). Furthermore, CCEs were compared between patients and controls in Mann-Whitney $U$-Tests two-tailed. Additionally, single case analyses were conducted with a modified $t$-test (two-tailed) that allows for an individual's score to be compared against a control sample (Crawford \& Howell, 1998). This test was applied using the program SINGLIMS.EXE (Crawford \& Garthwaite, 2002).

For uncrossed different hemispace trials, and crossed same hemispace trials, in which visual and tactile cues are assumed to map onto different cerebral hemispheres, this procedure allowed for investigating whether AgCC patients require interhemispheric transfer via the corpus callosum for remapping modality-specific spatial representations into a supramodal frame of reference, or whether early reorganization mechanisms, e.g. in the anterior commissure, may compensate for the absence of the corpus callosum. If early reorganization mechanisms are sufficient for compensating for the absence of the corpus callosum, CCEs of the IE should not differ significantly between patients and controls. However, if reorganization mechanisms are not sufficient, we would expect reduced interactions between vision and touch in such trials. Furthermore, for uncrossed same hemispace trials, and crossed different hemispace trials, in which visual and tactile cues are assumed to map onto identical cerebral hemispheres, this procedure allowed for investigating whether the absence of the corpus callosum in AgCC patients alters information processing within one hemisphere. If information processing within one hemisphere is not affected, CCEs of the IE should not differ significantly between patients and controls. However, if intrahemispheric, cerebral functioning is affected by the absence of the corpus callosum, CCEs should differ significantly between the two groups of participants

Because Mann-Whitney $U$-Tests did not reveal significant group differences, we additionally applied a bootstrap resampling approach (Efron, 1979; Efron \& Tibshirani, 1993) to compare patients with controls. Bootstrapping was carried out by drawing 1000 random samples of size 3 (i.e., samples of the same group size as our patient group) from the pool of controls and calculating their mean CCEs of the IE for uncrossed and crossed same and different hemispace trials as well as for the differences between uncrossed and crossed same and different hemispace trials (uncrossed same hemispace trials minus uncrossed different hemispace trials; crossed same hemispace trials minus crossed different hemispace trials). This procedure created an empirical distribution of mean CCE values to which the patient data were compared. A significant difference of the patient population would be assumed if their mean fell within the $2.5 \%$ of the highest or lowest values of the bootstrap distribution, thus testing at the $5 \%$ level with two tails.

Furthermore, to estimate the statistical power of our study, we performed a power analysis using G*Power 3.1. (Faul, Erdfelder, Buchner, \& Lang, 2009).

\section{Results}

\subsection{Error rates, reaction times and inverse efficiency}

Means and standard errors are provided in Table 1. In uncrossed trial blocks, mean error rates, reaction times, and IE did not differ significantly between patients and controls for (1) incongruent same hemispace trials (error rate: $U=65, p=0.58$; reaction time: $U=64, p=0.56$; IE: $U=65.5, p=0.60)$, (2) congruent same

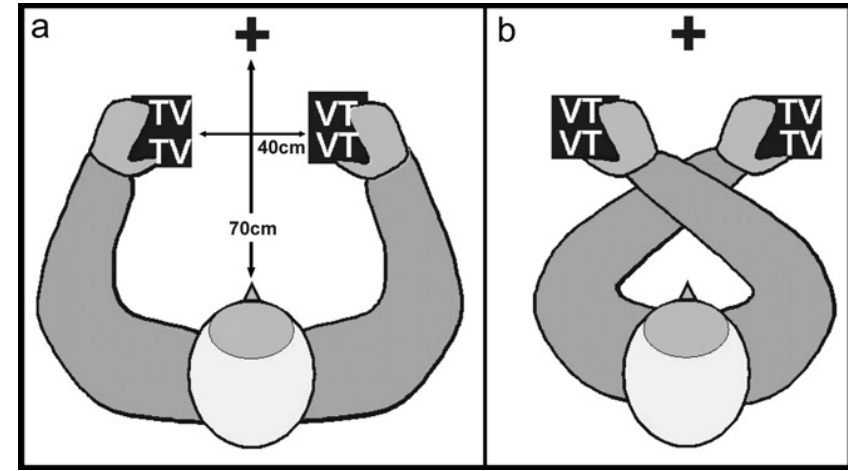

Fig. 2. Visuotactile crossmodal congruency task with (a) uncrossed and (b) crossed hands. A central fixation cross was displayed at a distance of $70 \mathrm{~cm}$, the distance between hands was $40 \mathrm{~cm}$. V, visual distractor; T, tactile target.

hemispace trials (error rate: $U=56, p=0.34$; reaction time: $U=58$, $p=0.40$; IE: $U=57.5, p=0.38)$, (3) incongruent different hemispace trials (error rate: $U=43, p=0.12$; reaction time: $U=75.5, p=0.94$; IE: $U=75.5, p=0.94)$. For (4) congruent different hemispace trials, reaction time $(U=76.5, p=0.98)$ and IE $(U=75.5, p=0.94)$ did not differ significantly between patients and controls. However, patients and controls differed in error rates for congruent different hemispace trials, with marginally-significantly larger error rates being found for patients (error rate: $U=38, p=0.08$ ).

For crossed trial blocks, the two groups of participants did not differ significantly in error rate, reaction time, and IE in any of the four conditions (incongruent different hemispace trials: error rate: $U=73, p=0.84$, reaction time: $U=72.5, p=0.82$, IE: $U=67.5, p=0.66$; congruent different hemispace trials: error rate: $U=76, p=0.96$, reaction time: $U=60.5, p=0.46$, IE: $U=56.5, p=0.36$; incongruent same hemispace trials: error rate: $U=59.5, p=0.42$, reaction time: $U=60.5, p=0.46$, IE: $U=59.5, p=0.42$; congruent same hemispace trials: error rate: $U=68.5, p=0.70$, reaction time: $U=65.5, p=0.60$, IE: $U=55.5, p=0.32$ ).

\subsection{Crossmodal congruency effects}

The effect of crossmodal interference on tactile judgements was assessed more directly by analyzing the crossmodal congruency effects (CCEs of the IE), i.e., the difference between incongruent and congruent conditions (see Table 1 for means and standard errors of patients and controls).

Table 1

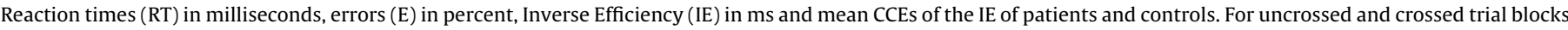

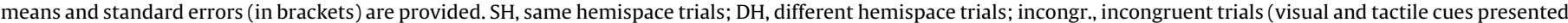
at different elevations), congr., congruent trials (visual and tactile cues presented at the same elevation).

\begin{tabular}{|c|c|c|c|c|c|c|c|}
\hline & & & & $E(\%)$ & $\mathrm{RT}(\mathrm{ms})$ & $\mathrm{IE}(\mathrm{ms})$ & CCE \\
\hline \multirow[t]{8}{*}{ Controls } & Uncrossed & $\mathrm{SH}$ & Incongr. & $15.6(2.8)$ & $414(19.9)$ & $507(31.0)$ & $83(13.1)$ \\
\hline & hands & & Congr. & $9.8(2.3)$ & $375(16.3)$ & $425(21.9)$ & \\
\hline & & $\mathrm{DH}$ & Incongr. & $12.1(2.4)$ & $396(17.8)$ & $460(24.0)$ & $21(11.7)$ \\
\hline & & & Congr. & $10(2.2)$ & $387(18.7)$ & $439(25.9)$ & \\
\hline & Crossed & $\mathrm{SH}$ & Incongr. & $10.8(2.1)$ & $402(19.6)$ & $459(24.7)$ & $-7(11.6)$ \\
\hline & hands & & Congr. & $11.7(2.3)$ & $389(18.6)$ & $465(29.9)$ & \\
\hline & & $\mathrm{DH}$ & Incongr. & $12.2(2.4)$ & $403(18.1)$ & $474(29.4)$ & $14(12.5)$ \\
\hline & & & Congr. & $12.3(2.1)$ & $398(19.4)$ & $460(24.2)$ & \\
\hline \multirow[t]{8}{*}{ Patients } & Uncrossed & $\mathrm{SH}$ & Incongr. & $16.3(4.8)$ & $382(46.2)$ & $453(39.6)$ & $88(18.1)$ \\
\hline & hands & & Congr. & $8.4(1.1)$ & $335(32.3)$ & $365(33.1)$ & \\
\hline & & $\mathrm{DH}$ & Incongr. & $13.4(2.2)$ & $386(40.6)$ & $445(44.5)$ & $10(12.3)$ \\
\hline & & & Congr. & $13.8(2.6)$ & $373(39.4)$ & $436(52.0)$ & \\
\hline & Crossed & $\mathrm{SH}$ & Incong. & $7.5(1.7)$ & $383(40.9)$ & $412(39.9)$ & $13(13.0)$ \\
\hline & hands & & Congr. & $9.7(3.0)$ & $363(37.1)$ & $399(31.6)$ & \\
\hline & & $\mathrm{DH}$ & Incongr. & $11.3(2.1)$ & $361(47.5)$ & $405(48.2)$ & $10(13.8)$ \\
\hline & & & Incongr. & $8.4(1.5)$ & $361(48.1)$ & $395(54.0)$ & \\
\hline
\end{tabular}




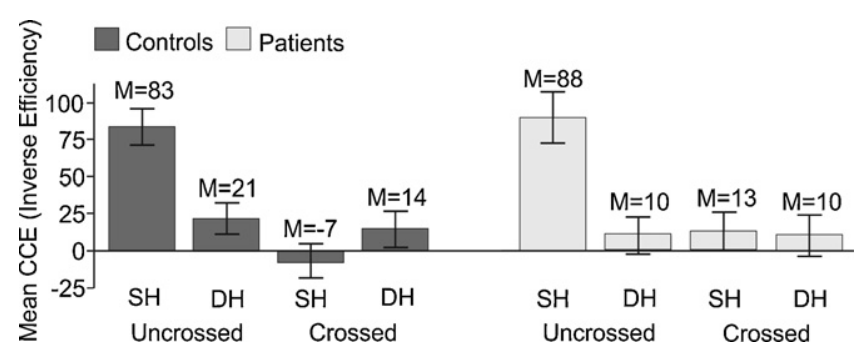

Fig. 3. CCEs of the inverse efficiencies. Shown are mean CCEs of patients and controls as well as individual scores of patients for same $(\mathrm{SH})$ and different hemispace trials (DH) with uncrossed and crossed hands. Error bars show standard error means.

Overall, the healthy controls' results resembled those reported in previous studies. With uncrossed hands, controls had larger CCEs for same hemispace trials compared to different hemispace trials $(t(30)=2.92, p=0.007)$, indicating a significant impact of visual distractors on tactile judgements in trials in which cues were presented at the same hand compared to trials in which visual cues were presented at different hands (Spence et al., 1998; Spence, Kingstone, et al., 2001; Spence et al., 2004).

With crossed hands, controls did not differ significantly in same and different hemispace trials $(t(30)=-0.01, p=0.66)$. Although previous studies have reported larger CCEs for same hemispace trials compared to different hemispace trials with crossed hands, again indicating a larger impact of visual cues that are presented at the same hand (Spence et al., 1998; Spence, Kingstone, et al., 2001; Spence et al., 2004), other studies suggest that crossing the hands can also lead to the intermediate effects we observed (Maravita et al., 2002; Spence \& Walton, 2005).

The comparison between patients and controls revealed that for uncrossed hand blocks, mean CCEs of the IE did not differ significantly between patients and controls for same (Mann-Whitney $U$-Test, two-tailed: $U=62.5, p=0.50$ ) as well as different hemispace trials $(U=67.5, p=0.66)$. Furthermore, CCEs of single patients did not differ significantly from controls for same (modified $t$ test, two-tailed: B.M.: $\mathrm{CCE}=158$, modified $t=1.02, p=0.32$; C.F.: $\mathrm{CCE}=58$, modified $t=-0.34, p=0.74$; M.K.: $\mathrm{CCE}=83$, modified $t=0.01, p=0.10$; P.N.: $\mathrm{CCE}=70$, modified $t=-0.18, p=0.86$; M.H.: $\mathrm{CCE}=70$, modified $t=-0.18, p=0.86$ ) and different hemispace trials (B.M.: CCE $=-14$, modified $t=-0.53, p=0.60$; C.F.: $\mathrm{CCE}=30$, modified $t=0.14, p=0.88$; M.K.: CCE $=-13$, modified $t=-0.51, p=0.62$; P.N.: CCE $=-1$, modified $t=-0.34, p=0.74$; M.H.: $\mathrm{CCE}=47$, modified $t=0.40, p=0.70$ ).

The same was found for crossed hand blocks. Again, mean CCEs did not differ significantly between patients and controls for different $(U=54.5, p=0.30)$, and same hemispace trials $(U=74.5, p=0.90)$. Also, CCEs of single patients did not differ significantly from controls for different (modified $t$-test, twotailed: B.M.: $\mathrm{CCE}=32$, modified $t=0.59, p=0.56$; C.F.: $\mathrm{CCE}=-8$, modified $t=-0.02, p=0.98$; M.K.: $C C E=41$, modified $t=0.72$, $p=0.48$; P.N.: $\mathrm{CCE}=26$, modified $t=0.50, p=0.62$; M.H.: $\mathrm{CCE}=-27$, modified $t=-0.31, p=0.76$ ) as well as same hemispace trials (B.M.: $\mathrm{CCE}=25$, modified $t=0.15, p=0.88$; C.F.: $\mathrm{CCE}=-6$, modified $t=-0.29, p=0.78$; M.K.: CCE $=-32$, modified $t=-0.66, p=0.52$; P.N.: CCE $=14$, modified $t=0.01, p=0.10$; M.H.: $\mathrm{CCE}=-49$, modified $t=0.49, p=0.62)$. Fig. 3 summarizes results of patients and controls.

\subsection{Bootstrap resampling}

Because non-significant results as assessed with the Mann-Whitney $U$-Test may partly be due to the small group size of patients, bootstrap resampling was applied to the CCEs of the IE of the control group for uncrossed and crossed same and different hemispace trials and for the difference between uncrossed and crossed same and different hemispace trials, and the patient data were compared to the bootstrapping results. These tests confirmed the results obtained with $U$-Tests. All CCE patient means clearly fell within the 95\% range of CCE mean distributions of the control group (see Figs. 4 and 5), indicating that the patient group did not differ from controls with respect to crossmodal visuotactile interference for both same and different hemispace conditions.

\subsection{Power analysis}

To estimate the statistical power we achieved with our sample, we performed a power analysis using $\mathrm{G}^{*}$ Power 3.1 (Faul et al., 2009). With sample sizes of $n=5$ (patients) and $n=31$ (controls) and an alpha of 0.05 we achieved a power of 0.5 , with magnitude equaling Hedges' $g=1$. Our study thus indicates that any difference between patients and controls would be expected to be smaller than $g=1.4$.

\section{Discussion}

The aim of the present study was to investigate possible changes in multisensory information processing in the congenitally acallosal brain. In a visuotactile crossmodal congruency task, we found that AgCC patients and healthy controls performed comparably in uncrossed different hemispace and crossed same hemispace trials (both presumably requiring interhemispheric processing) as well as uncrossed same hemispace and crossed different hemispace trials (both presumably requiring intrahemispheric processing). In congruent as well as incongruent same and different hemispace trials with uncrossed and crossed hands, patients and controls did not differ in reaction times, error rates, or IE. Furthermore, the impact of visual cues on the perception of touch, measured in terms of the CCE of IE, did not differ between the two groups of participants. Since this was also true for trials in which patients' hands crossed the vertical visual meridian and stimuli were presented at the same hand, our results are markedly different from those obtained in a split brain patient by Spence, Kingstone, et al. (2001). In the split brain patient, the authors observed a failure to remap visual space to the current hand position in the crossed hand posture and suggested that the corpus callosum is crucial for the maintenance of an intact representation of visuotactile space that the brain usually recruits the corpus callosum for purposes of spatial remapping is corroborated by the finding that monkeys were unable to remap visual information between hemispheres after callosotomy (Berman, Heiser, Saunders, \& Colby, 2005; Heiser, Berman, Saunders, \& Colby, 2005). However, because AgCC patients performed comparably to controls, this conclusion cannot be applied to the congenitally acallosal brain. Instead, we suggest that the absence of callosal fibres can be compensated for, allowing for an intact processing of visuotactile information when the corpus callosum is developmentally absent. Thus, an early onset of compensatory mechanisms may allow for an adaptation of interhemispheric processing in the absence of the corpus callosum in the congenitally acallosal, but not in the surgically disconnected split brain.

With respect to the function of the intact corpus callosum, the finding that information processing was comparable in trials that presumably involve interhemispheric processing (uncrossed different hemispace trials and crossed same hemispace trials) is particularly remarkable: In healthy individuals, more than 200 million callosal fibres transfer information from one hemisphere to the other, and integrate information between the hemispheres (Aboitiz et al., 1992a, 1992b; Bloom \& Hynd, 2005). Furthermore, multisensory information processing is importantly different 

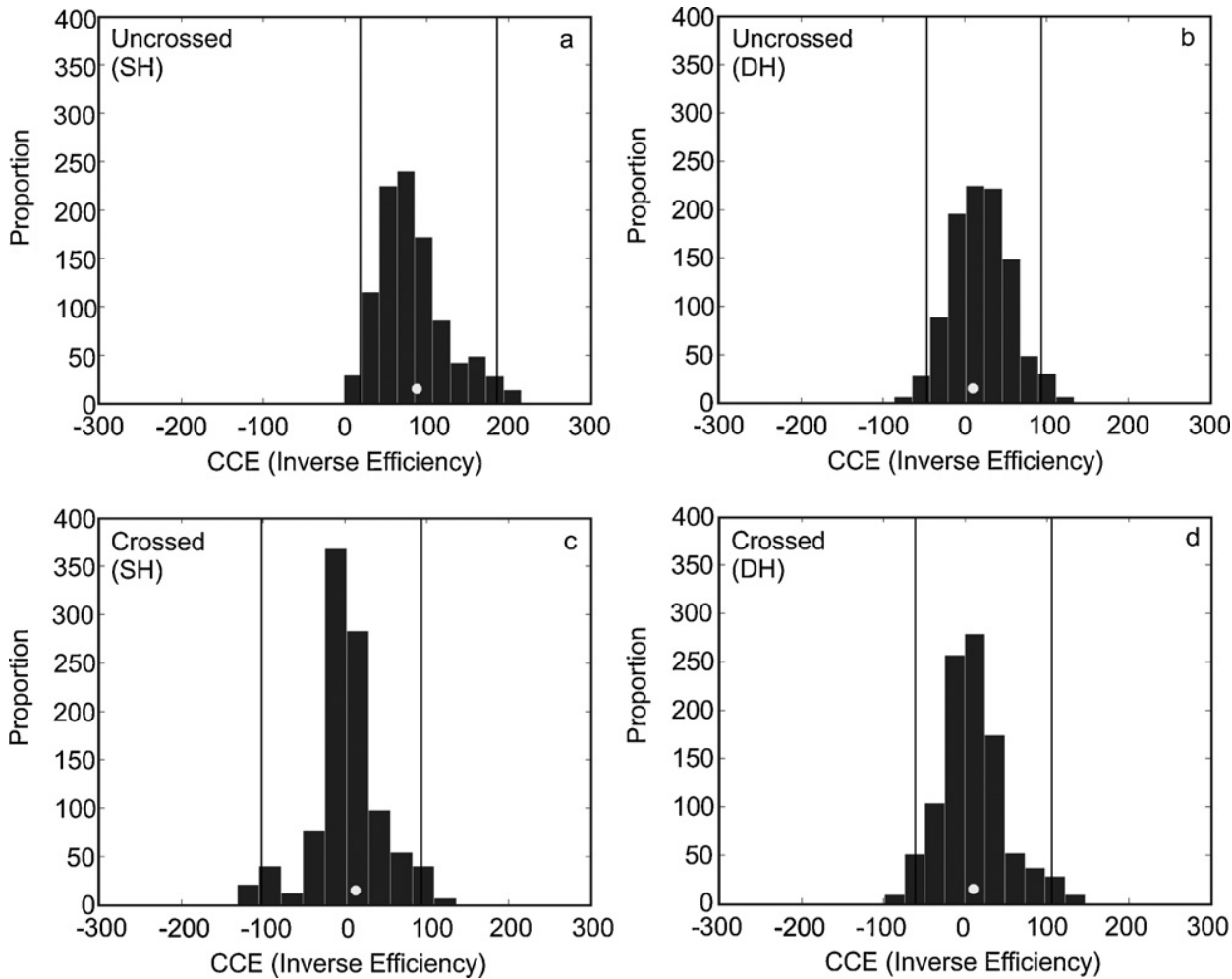

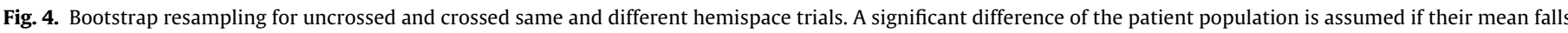

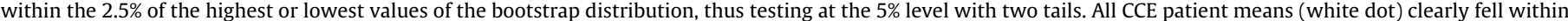
the normal range of CCE mean distributions of the control group. SH, same hemispace trials; $\mathrm{DH}$, different hemispace trials. Vertical lines show the $2.5 \%$ cut-off points.

from unisensory processing, which is due to the fact that sensory information from the different senses must be remapped within 100-150 ms (Heed et al., 2010) into common coordinates in order to derive a unified sensory experience (Angelaki, Gu, \& Deangelis, 2009; Bulkin \& Groh, 2006; Driver \& Noesselt, 2008; McGuire \& Sabes, 2009; Morrell, 1972).

Possibly, plasticity of the anterior commissure - which was enlarged in four patients (P.N., B.M., C.F., M.H.) - allows for early compensation for the absence of the corpus callosum in trials that require interhemispheric processing. This is in line with Guenot (1998), who outlined that information transfer via the anterior commissure is the most important compensatory mechanism in patients with AgCC. Indeed, only a small number of fibres are necessary to ensure information transmission adequate for most split brain tests (Chiarello, 1980). However, due to the important difference between field of origin of callosal and anterior commissure fibres, the compensation capacity of the anterior commissure is probably limited, although termination fields of anterior commissure fibres may be enlarged in patients with AgCC compared to healthy individuals. This suggests that further mechanisms must be involved, e.g. an increased use of ipsilateral pathways that then connect to commissural systems (Cao et al., 1994; Chiarello, 1980).

Furthermore, bimodal cells, which integrate information from different sensory modalities (reviewed by Stein \& Stanford, 2008), are not only found in cortical structures (e.g. Graziano, Yap, \& Gross, 1994; Wallace, Meredith, \& Stein, 1992). Therefore, several non-cortical opportunities for interhemispace interactions exist, which may explain our results, e.g. the colliculopulvinar pathway,
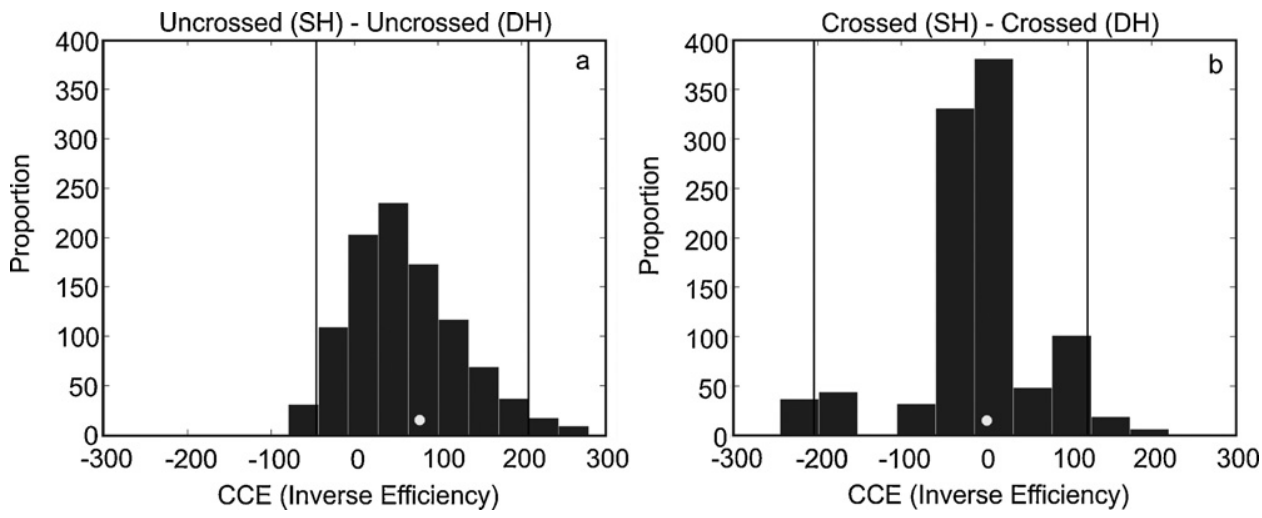

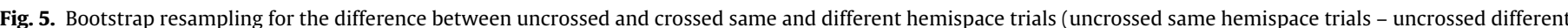

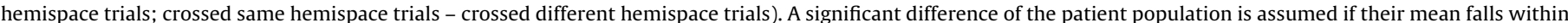

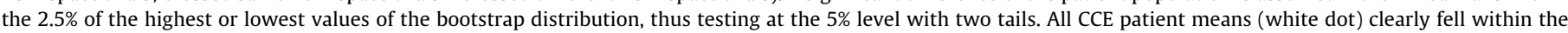
normal range of CCE mean distributions of the control group. SH, same hemispace trials; DH, different hemispace trials. Vertical lines show the $2.5 \%$ cut-off points. 
habenular commissure, posterior commissure, and intercollicular commissure. For instance, such bimodal cells are found in the putamen (e.g. Graziano \& Gross, 1993), and superior colliculus and could result from transfer along the commissura posterior and tectalis. Also, there is some evidence from studies with split brain macaques that spatial representations in the parietal cortex can be updated without use of direct cortico-cortical links, although pathways for across-hemifield updating seem to be less effective (Heiser et al., 2005).

In addition to trials involving interhemispheric processing of visuotactile information, we investigated trials which presumably require intrahemispheric processing (uncrossed same hemispace trials and crossed different hemispace trials). For instance, AgCC is not only associated with the absence of a major pathway, but also with the presence of a novel one: "Probst bundles", anteriorposterior paths of misrouted callosal fibres that failed to cross the midline during prenatal brain development and remain within the ipsilateral hemisphere, are a common feature in patients with AgCC (Hetts et al., 2006; Utsunomiya, Yamashita, Takano, \& Okazaki, 2006). Indeed, an in vitro examination revealed that Probst bundles can maintain functionality, become myelinated, and survive into old age (Lefkowitz, Durand, Smith, \& Silver, 1991). However, we found that effects of visual cues on the perception of touch were neither increased nor reduced in trials involving intrahemispheric processing. Thus, Probst bundles or other, unknown brain alterations do not seem to have affected visuotactile information processing within one hemisphere.

Taken together, our results show that AgCC patients do not perform differently from healthy controls in a visuotactile interference task. Therefore, we assume that developmental brain plasticity allows for early compensatory changes which provide multisensory capacities that require an intact corpus callosum in healthy individuals.

\section{Acknowledgements}

\section{Supported by the DFG through GZ: GU227/11-1}

\section{References}

Aboitiz, F., Scheibel, A. B., Fisher, R. S. \& Zaidel, E. (1992a). Fibre composition of the human corpus callosum. Brain Research, 598, 143-153.

Aboitiz, F., Scheibel, A. B., Fisher, R. S. \& Zaidel, E. (1992b). Individual differences in brain asymmetries and fibre composition in the human corpus callousm. Brain Research, 598, 154-161.

Angelaki, D. E., Gu, Y. \& Deangelis, G. C. (2009). Multisensory integration: Psychophysics, neurophysiology, and computation. Current Opinion in Neurobiology, 19, 452-458.

Bavelier, D., Levi, D. M., Li, R. W., Dan, Y. \& Hensch, T. K. (2010). Removing brakes on adult brain plasticity: From molecular to behavioral interventions. Journal of Neuroscience, 30, 14964-14971.

Bedeschi, M. F., Bonaglia, M. C., Grasso, R., Pellegri, A., Garghentino, R. R., Battaglia M. A., et al. (2006). Agenesis of the corpus callosum: Clinical and genetic study in 63 young patients. Neurology, 34, 186-193.

Berman, R. A., Heiser, L. M., Saunders, R. C. \& Colby, C. L. (2005). Dynamic circuitry for updating spatial representations. I. Behavioral evidence for interhemispheric transfer in the split-brain macaque. Journal of Neurophysiology, 18, 3228-3248.

Bloom, J. S. \& Hynd, G. W. (2005). The role of the corpus callosum in interhemispheric transfer of information: Excitation or inhibition? Neuropsychology Review, 15 59-71.

Brown, W. S., Jeeves, M. A., Dietrich, R. \& Burnison, D. S. (1999). Bilateral field advantage and evoked potential interhemispheric transmission in commissurotomy and callosal agenesis. Neuropsychologia, 37, 1165-1180.

Brown, W. S., Thrasher, E. D. \& Paul, L. K. (2001). Interhemispheric Stroop effects in partial and complete agenesis of the corpus callosum. Journal of the International Neuropsychological Society, 7, 302-311.

Bulkin, D. A. \& Groh, J. M. (2006). Seeing sounds: Visual and auditory interactions in the brain. Current Opinion in Neurobiology, 16, 415-419.

Cao, Y., Vikingstad, E. M., Huttenlocher, P. R., Towle, V. L. \& Levin, D. N. (1994) Functional magnetic resonance studies of the reorganization of the human hand sensorimotor area after unilateral brain injury in the perinatal period. Proceedings of the National Academy of Sciences, 91, 9612-9616.
Chiarello, C. (1980). A house divided? Cognitive functioning with callosal agenesis. Brain and Language, 11, 128-158.

Crawford, J. R. \& Garthwaite, P. H. (2002). Investigation of a single case in neuropsychology. Confidence limits on the abnormality of test scores and test score differences. Neuropsychologia, 40, 1196-1208.

Crawford, J. R. \& Howell, D. C. (1998). Comparing an individual's test score against norms derived from small samples. The Clinical Neuropsychologist, 12, 482-486.

Di Pellegrino, G., Làvadas, E. \& Farné, A. (1997). Seeing where your hands are. Nature, $388,730$.

Di Virgilio, G., Clarke, S., Pizzolato, G. \& Schaffner, T. (1999). Cortical regions contributing to the anterior commissure in man. Experimental Brain Research, 124, $1-7$.

Driver, J. \& Noesselt, T. (2008). Multisensory interplay reveals crossmodal influences on 'sensory-specific' brain regions, neural responses, and judgements. Neuron, $57,11-23$.

Duffau, H. (2006). Brain plasticity: From pathophysiological mechanisms to therapeutic applications. Journal of Clinical Neuroscience, 13, 885-897.

Efron, B. \& Tibshirani, R. J. (1993). An introduction to the bootstrap. New York: Chapman \& Hall

Efron, B. (1979). Bootstrap methods: Another look at the jackknife. Annals of Statistics, 7, 1-26.

Faul, F., Erdfelder, E., Buchner, A. \& Lang, A. (2009). Statistical power analysis using $\mathrm{G}^{*}$ Power 3.1: Test for correlation and regression analysis. Behaviour Research Methods, 41, 1149-1160.

Friefeld, S., MacGregor, D., Chuang, S. \& Saint-Cyr, J. (2000). Comparative study of inter- and intrahemispheric somatosensory functions in children with partial and complete agenesis of the corpus callosum. Developmental Medicine and Child Neurology, 4, 831-838.

Gage, F. H. (2004). Structural plasticity of the adult human brain. Dialogues in Clinical Neuroscience, 6, 135-141.

Gazzaniga, M. S. (1995). Principles of human brain organization derived from splitbrain studies. Neuron, 14, 217-228.

Gazzaniga, M. S. (2005). Forty-five years of split-brain research and still going strong. Nature Reviews Neuroscience, 6, 653-659.

Graziano, M. S. A. \& Gross, C. G. (1993). A bimodal map of space: Somatosensory receptive fields in the macaque putamen with corresponding visual receptive fields. Experimental Brain Research, 97, 96-109.

Graziano, M. S. A., Yap, G. S. \& Gross, C. G. (1994). Coding visual space by premotor neurons. Science, 266, 1054-1057.

Guenot, M. (1998). Interhemispheric transfer and agenesis of the corpus callosum: Capacities and limitations of the anterior commissure. Neurochirurgie, 44 , 113-115.

Guerri, C., Pascual, M. \& Renau-Piqueras, J. (2001). Glia and fetal alcohol syndrome. NeuroToxicology, 22, 593-599.

Heed, T., Habets, B., Sebanz, N. \& Knoblich, G. (2010). Others' actions reduce crossmodal interaction in peripersonal space. Current Biology, 20, 1345-1349.

Heiser, L. M., Berman, R. A., Saunders, R. C. \& Colby, C. L. (2005). Dynamic circuitry for updating spatial representations. II. Physiological evidence for interhemispheric transfer in area LIP of the split-brain macaque. Journal of Neurophysiology, 94, 3249-3258.

Hetts, S. W., Sherr, E. H., Gobuty, S., Chao, S. \& Barkovich, A. J. (2006). Anomalies of the corpus callosum: MR analysis of the phenotypic spectrum. American Journal of Roentgenology, 187, 1343-1348.

Hicks, S. P. \& D`Amato, C. J. (1970). Motor-sensory and visual behavior after hemispherectomy in newborn and mature rats. Experimental Neurology, 29, 416-438.

Hines, R. J., Paul, L. K. \& Brown, W. S. (2002). Spatial attention in agenesis of the corpus callosum: Shifting attention between visual fields. Neuropsychologia, 40, 1804-1814.

Jeeves, M. A., Silver, P. H. \& Jacobson, I. (1988). Bimanual coordination in callosal agenesis and partial commissurotomy. Neuropsychologia, 26, 833-850.

Johnston, M. V. (2004). Clinical disorders of brain plasticity. Brain E' Development, $26,73-80$.

Johnston, M. V., Ishida, A., Ishida, W. N., Matsushita, H. B., Nishimura, A. \& Tsuji, M. (2009). Plasticity and injury in the developing brain. Brain \& Development, 31, $1-10$.

Kleim, J. A. \& Jones, T. A. (2008). Principles of experience-dependent neural plasticity: implications for rehabilitation after brain damage. Journal of Speech, Language, and Hearing Research, 51, 225-239.

Klinger, J. \& Gloor, P. (1960). The connections of the amygdala and of the anterior temporal cortex in the human brain. Journal of Comparative Neurology, 115, 333-369.

Kolb, B., Gibb, R. \& Robinson, T. E. (2003). Brain plasticity and behavior. Current Directions in Psychological Science, 12, 1-5.

Lassonde, M., Sauerwein, H., Chicoine, A. \& Geoffroy, G. (1991). Absence of disconnexion syndrome in callosal agenesis and early callosotomy: Brain reorganization or lack of structural specificity during ontogeny? Neuropsychologia, 29, 481-495.

Lefkowitz, M., Durand, D., Smith, G. \& Silver, J. (1991). Electrical properties of axons within probst bundles of acallosal mice and callosi that have reformed upon glial-coated polymer implants. Experimental Neurology, 113, 306-314.

Lassonde, M., Sauerwein, H., McCabe, N., Laurencelle, L. \& Geoffroy, G. (1988). Extents and limits of cerebral adjustment to early section or congenitally absence of the corpus callosum. Behavioural Brain Research, 30, 165-181.

Lehrl, S. (1977). Mehrfachwahl-Wortschatz-Intelligenztest MWT-B. Straube: Erlangen. 
Maravita, A., Spence, C., Kennett, S. \& Driver, J. (2002). Tool-use changes multimodal spatial interactions between vision and touch in normal humans. Cognition, 38 , B25-B34.

McGuire, L. M. M. \& Sabes, P. N. (2009). Sensory transformations and the use of multiple reference frames for reach planning. Nature Neuroscience, 12, 1056-1061.

Mooshagian, E., Iacoboni, M. \& Zaidel, E. (2009). Spatial attention and interhemispheric visuomotor integration in the absence of the corpus callosum. Neuropsychologia, 47, 933-937.

Morrell, F. (1972). Visual system's view of acoustic space. Nature, 238, 44-46.

Mueller, K. L. O., Marion, S. D., Paul, L. K. \& Brown, W. S. (2009). Bimanual motor coordination in agenesis of the corpus callosum. Behavioural Neuroscience, 123 1000-1011.

Oldfield, R. C. (1971). The assessment and analysis of handedness: The Edinburgh Inventory. Neuropsychologia, 9, 56-64.

Paul, L. K., Brown, W. S., Adolphs, R., Tyszka, J. M., Richards, L. J., Mukherjee, P. \& Sherr, E. H. (2007). Agenesis of the corpus callosum: Genetic, developmental and functional aspects of connectivity. Nature Reviews Neuroscience, 8, 287-299.

Pavani, F., Spence, C. \& Driver, J. (2000). Visual capture of touch: Out-of-the-body experiences with rubber gloves. Psychological Science, 11, 353-359.

Poffenberger, A. T. (1912). Reaction time to retinal stimulation with special reference to the time lost in conduction through nerve centres. Archives of Psychology, 23, $1-73$.

Ramachandran, V. S. (1993). Behavioral and magnetoencephalography correlates of plasticity in the adult human brain. Proceedings of the National Academy of Sciences, 90, 10413-10420.

Riley, E. P., Mattson, S. N., Sowell, E. R., Jernigan, T. L., Sobel, D. F. \& Jones, K. L. (1995). Abnormalities of the corpus callosum in children prenatally exposed to alcohol. Alcoholism, Clinical and Experimental Research, 19, 1198-1203.

Röder, B., Kusmierek, A., Spence, C. \& Schicke, T. (2007). Developmental vision determines the reference frame for multisensory control of action. Proceedings of the National Academy of Science, 104, 4753-4758.

Sauerwein, H. C., Lassonde, M. C., Cardu, B. \& Geoffroy, G. (1981). Interhemispheric integration of sensory and motor functions in agenesis of the corpus callosum. Neuropsychologia, 19, 445-454.

Schell-Apacik, C. C., Wagner, K., Bihler, M., Ertl-Wagner, B., Heinrich, U., Klopocki, E. et al. (2008). Agenesis and dysgenesis of the corpus callosum: Clinical, genetic and neuroimaging findings in a series of 41 patients. American Journal of Medical Genetics Part A, 146, 2501-2511.

Schicke, T., Bauer, F. \& Röder, B. (2009). Interactions of different body parts in peripersonal space: How vision of the foot influences tactile perception of the hand. Experimental Brain Research, 192, 703-715.

Seymour, S. A., Reuter-Lorenz, P. A. \& Gazzaniga, M. S. (1994). The disconnection syndrome: Basic findings reaffirmed. Brain, 117, 105-115.

Silver, P. H. \& Jeeves, M. A. (1994). Motor coordination in agenesis of the corpus callosum. In M. Lassonde, \& M. A. Jeeves (Eds.), Callosal agenesis: A natural split brain? (pp. 207-219). New York: Plenum Press.

Spence, C., Kingstone, A., Shore, I. D. \& Gazzaniga, M. S. (2001). Representation of visuotactile space in the split brain. Psychological Science, 12, 90-93.

Spence, C., Pavani, F. \& Driver, J. (1998). What crossing the hands can reveal about crossmodal links in spatial attention. Abstracts of the Psychonomic Society, 3 , 13.

Spence, C., Pavani, F. \& Driver, J. (2004). Spatial constraints on visual-tactile cross-modal distractor congruency effects. Cognitive, Affective, \&'Behavioral Neuroscience, 4, 148-169.

Spence, C., Shore, D. I., Gazzaniga, M. S., Soto-Faraco, S. \& Kingstone, A. (2001). Failure to remap visuotactile space across the midline in the split-brain. Canadian Journal of Experimental Psychology, 55, 133-140.

Spence, C. \& Walton, M. (2005). On the inability to ignore touch when responding to vision in the crossmodal congruency task. Acta Psychologica, 118, 47-70.

Sperry, R. W., Gazzaniga, M. \& Bogen, J. E. (1969). Interhemispheric relationships: The neurocortical commissures: Syndrome of hemispheric disconnection. In P. J. Vinken, \& G. W. Bruyn (Eds.), Handbook of clinical neurology (pp. 273-290). Amsterdam: Elsevier.

Stein, B. E. \& Stanford, T. R. (2008). Multisensory integration: Current issues from the perspective of a single neuron. Nature Reviews Neuroscience, 9, 255-266.

Utsunomiya, H., Yamashita, S., Takano, K. \& Okazaki, M. (2006). Arrangement of fibre tracts forming probst bundle in complete callosal agenesis: Report of two cases with an evaluation by diffusion tensor tractography. Acta Radiologica, 47, 1063-1066.

Wallace, M. T., Meredith, M. A. \& Stein, B. E. (1992). Integration of multiple sensory inputs in the cat cortex. Experimental Brain Research, 91, 484-488. 